\title{
Evidence for T Tauri-like emission in the EXor V1118 Ori from near-IR and X-ray data ${ }^{\star}$
}

\author{
D. Lorenzetti ${ }^{1}$, T. Giannini ${ }^{1}$, L. Calzoletti ${ }^{1,2}$, S. Puccetti $^{1,3}$, S. Antoniucci ${ }^{1,3}$, A. A. Arkharov ${ }^{4}$, A. Di Paola ${ }^{1}$, \\ V. M. Larionov ${ }^{5}$, and B. Nisini ${ }^{1}$ \\ 1 INAF - Osservatorio Astronomico di Roma, via Frascati 33, 00040 Monte Porzio, Italy \\ e-mail: [dloren;giannini;calzol; puccetti; antoniucci;dipaola;nisini]@mporzio.astro.it \\ 2 Università degli Studi di Cagliari - Dipartimento di Fisica, S.P. Monserrato-Sestu Km 0.700, 09042 Monserrato-CA, Italy \\ 3 Università degli Studi di Roma "Tor Vergata" - Dipartimento di Fisica, via della Ricerca Scientifica 1, 00133 Roma, Italy \\ ${ }^{4}$ Central Astronomical Observatory of Pulkovo, Pulkovskoe shosse 65, 196140 St. Petersburg, Russia \\ e-mail: arkharov@mail.ru \\ 5 Astronomical Institute of St. Petersburg University, Russia \\ e-mail: vml@vL1104.spb.edu
}

Received 21 November 2005 / Accepted 28 February 2006

\section{ABSTRACT}

\begin{abstract}
Aims. We present a near-IR study of the EXor variable V1118 Ori, performed by following a slightly declining phase after a recent outburst. In particular, the near-IR $(0.8-2.3 \mu \mathrm{m})$ spectrum, obtained for the first time, shows a wide variety of emission features of the $\mathrm{H}$ I (Paschen and Brackett series), He I recombination, and $\mathrm{CO}$ overtone.

Methods. By comparing the observed spectrum with a wind model, a mass loss rate of $4 \times 10^{-8} M_{\odot} \mathrm{yr}^{-1}$ can be derived along with other parameters whose values are typical of an accreting T Tauri star. In addition, we have used X-ray data from the XMM archive, taken in two different epochs during the declining phase monitored in IR. The X-ray emission (in the range $0.5-10 \mathrm{keV}$ ) permits us to derive several parameters (as plasma temperatures and $L_{\mathrm{X}}$ luminosity) that confirm the T Tauri nature of the source.

Results. In the near-IR, the object maintains a low extinction $\left(A_{\mathrm{V}} \lesssim 2\right)$ during all the activity phases, confirming that variable extinction does not contribute to brightness variations. The lack of both a significant amount of circumstellar material and any evidence of IR cooling from collimated jet/outflow driven by the source indicates that at least this member of the EXor class is in a late stage of the pre-main sequence evolution. When going from inactive to active phases, the luminosity increases considerably (from $1.4 L_{\odot}$ to more than $25 L_{\odot}$ ) and the observed spectral energy distribution assumes different shapes, all typical of a T Tauri star. In the $\mathrm{X}$-ray regime, an evident fading is present, detected in the post-outburst phase, which cannot be reconciled with the presence of any absorbing material. This circumstance, combined with the persistence (in the pre- and post-outburst phases) of a temperature component at about $10 \mathrm{MK}$, suggests that accretion has some influence in regulating the coronal activity.
\end{abstract}

Key words. stars: emission line - stars: pre-main sequence - stars: variables: general - stars: individual: V1118 Ori X-rays: stars

\section{Introduction}

The star V1118 Ori is considered a pre-main sequence EXor variable that undergoes subsequent outbursts in optical light. The EXor class (whose name stems from the prototype EX Lup) is composed of nine members as defined and listed by Herbig (1989). These sources are characterised phenomenologically by repetitive outbursts with an amplitude of up to $5 \mathrm{mag}$, lasting one year or less, and with a recurrence time of about 5-10 years. They are supposed to experience an intermediate phase between the strong FUor eruptions and the more quiescent main sequence (Herbig 1977; Hartmann et al. 1993), during which they exhibit a lower activity level than the FUor one.

The differences between FUor and EXor outbursts are not so evident in intensity, since both classes have comparable outburst amplitudes, but they do differ in duration, as the former is bright for decades and the latter only for months. Indeed, whether the EXor evolutionary stage follows the FUor one or merely is a less evident manifestation of the same phase has not

* Based on observations collected at the AZT-24 telescope (Campo Imperatore, Italy). yet been completely ascertained. As for the FUor analogs, the EXor outbursts are believed to arise from enhanced accretion events from a circumstellar disk, although associated with lesser values of the mass accretion rate $(\dot{M})$. This very uncertain picture that emerges when interpreting the EXor phenomenum stems from the small number of known objects and from the lack of a long-term multi-frequency monitoring of the photometric and spectroscopic properties.

By examining the case of the eruptive object V1118 Ori, four outbursts have been observed so far, although archival plates suggest that there were other flares in the past (1939-1956 Paul et al. 1995). The first documented burst was during the period 1982-1984 (Chanal 1983; Parsamian \& Gasparian 1987), the second in 1988-1990 (Parsamian et al. 1993), the third in 1992-1994 (García García et al. 1995), and the fourth in 1997-1998 (Hayakawa et al. 1998; García García \& Parsamian 2000). The last outburst at the end of 2004 was announced by Williams \& Heathcote (2005) and currently (September 2005), the object is slowly fading but has not yet reached its quiescent phase. Typically, the amplitude of the outburst (in $V$ band) is larger than 3 mag (from about 17-18 to 14.5) and the duration is 
longer than 2 years, with a rise time of $8-10$ months followed by a long-lived decline phase of about 1.5 years. An optical spectrum (from 3900 to $5600 \AA$ ) was obtained (Gasparian et al. 1990) during the rising period of the second outburst in the early 1989. It shows emission lines of H I, Ca II, Fe I, and Fe II, and the presence of P-Cyg profiles in the Balmer series is suspected: this spectrum is similar to that of $\mathrm{T}$ Tauri stars with moderate intensity. Prior to the most recent outburst, the source was photometrically observed at near-IR wavelengths only three times during both outburst and quiescence phases.

We present here the first near-IR spectrum of V1118 Ori along with broad and narrow band imaging data obtained during the last declining phase. A newly obtained X-ray detection during the same phase is presented as a result of a search in the existing archives. Accretion processes have been suggested as the possible origin of the X-ray emission of young low mass objects (e.g. Shu et al. 1997). However, the mechanisms are still unclear since many of these objects emit X-rays via solar-like magnetically trapped coronal plasma (see the review by Feigelson \& Montmerle 1999; Kastner et al. 2004a).

Archival X-ray data come from a multi-wavelength campaign to monitor the 2004/2005 outburst of V1118 Ori. Such monitoring is conducted by Audard et al. (2005), who have very recently presented their first results. They focus mainly on the X-ray properties, although accurately sampled optical and IR light curves are presented, as well. They argue that the X-ray emission was due to a corona, but it was influenced by the accretion during the outburst. Their time coverage partially overlaps with our early observations that complement their results by covering a subsequent period, which is relevant for ascertaining the source behaviour.

The IR study carried out during the declining/quiescent phase allows us to investigate how the properties of the circumstellar matter prior to the outburst will influence the accretion through the outburst itself. The same study during the active phase samples how the circumstellar material is altered by intermittent mass loss. The X-ray investigation carried out during an optical/near-IR monitoring can help in discriminating between the mechanism(s) that generate(s) the high energy emission in low mass protostars. Our aim is manifold: (i) to better understand how the IR spectral signatures are related to the intrinsic nature of an EXor, (ii) to derive a consistent picture for both the IR and X-ray behaviour, and (iii) to build up a systematic IR database useful as a reference for the next monitoring of the quiescence and subsequent outburst phases. After a short presentation in Sect. 2 of our observations and data reduction procedures, we provide and discuss the results in Sect. 3, giving our conclusions in Sect. 4.

\section{Observations and data reduction}

\subsection{Near-IR imaging and spectroscopy}

Near-IR data were obtained at the $1.1 \mathrm{~m}$ AZT-24 telescope located at Campo Imperatore (L'Aquila - Italy) equipped with the imager/spectrometer SWIRCAM (D'Alessio et al. 2000), which is based on a $256 \times 256 \mathrm{HgCdTe}$ PICNIC array. Photometry is performed with broad band filters $J(1.25 \mu \mathrm{m}), H(1.65 \mu \mathrm{m})$, and $K(2.20 \mu \mathrm{m})$, along with a narrow band one, centred at $2.122 \mu \mathrm{m}$ on the $1-0 S(1) \mathrm{H}_{2}$ transition. The total field of view is $4.4 \times 4.4 \operatorname{arcmin}^{2}$, which corresponds to a plate scale of $1.04 \mathrm{arcsec} / \mathrm{pixel}$. Low resolution $(\mathcal{R} \sim 250)$ spectroscopy is obtained by means of two IR grisms $G_{\text {blue }}$ and $G_{\text {red }}$ covering the $Z J$ $(0.83-1.34 \mu \mathrm{m})$ and $H K(1.44-2.35 \mu \mathrm{m})$ bands, respectively, in
Table 1. Journal of observations.

\begin{tabular}{cccc}
\hline \hline UT Date & MJD $^{a}$ & Mode & Filter/Grism \\
\hline Mar. 20, 05 & 53449 & Phot & $J, K$ \\
Apr. 03, 05 & 53463 & Phot & $J, K$ \\
Apr. 15, 05 & 53475 & Phot & $J, K$ \\
Sep. 06, 05 & 53619 & Phot & $J, K, \mathrm{H}_{2}$ \\
Sep. 11, 05 & 53624 & Phot/Spec & $J H K, G_{\mathrm{b}}, G_{\mathrm{r}}$ \\
\hline
\end{tabular}

${ }^{a} \mathrm{MJD}=$ modified Julian Date.

two subsequent exposures. The long slit is not orientable in position angle, and it samples a pre-defined portion of the focal plane, $2 \times 260 \operatorname{arcsec}^{2}$ in the east-west direction. Details of the observations are given in Table 1 . All the observations were obtained by dithering the telescope around the pointed position. The raw imaging data were reduced by using standard procedures for bad pixel removal, flat fielding, and sky subtraction. Continuum-free images in the narrow band filter were obtained as a first step by subtracting an appropriately scaled $K$ image from the $\mathrm{H}_{2}$ image. Such scaling has been obtained by performing the photometry of a number of stars located in different positions within the field.

Long slit spectroscopy was carried out in the standard $\mathrm{ABB}^{\prime} \mathrm{A}^{\prime}$ mode with a total integration time of $800 \mathrm{~s}$. The observations were flat-fielded, sky-subtracted, and corrected for the optical distortion in both the spatial and spectral directions. Telluric features were removed by dividing the extracted spectra by that of a normalized telluric standard star, once corrected for its intrinsic spectral features. Wavelength calibration was derived from the $\mathrm{OH}$ lines present in the raw spectral images, while flux calibration was obtained from our photometric data.

\subsection{X-ray observations}

To obtain reliable X-ray data on V1118 Ori, we searched the public archives and found the XMM-Newton results of pointed observations taken during our monitoring period. The relevant information on both the X-ray telescope and EPIC (PN and MOS) cameras are given by Jansen et al. (2001), Strüder et al. (2001), and Turner et al. (2001), respectively. Table 2 summarises the two XMM-Newton observations available. The first was obtained a few months after the most recent outburst (see also Audard et al. 2005), while the second (on Sep. 08, 2005), obtained 7 months later, is almost simultaneous with our near-IR spectrum, taken 3 days later. The data have been processed using the XMM-Newton Science Analysis Survey (SAS) v.6.1.0. We used the event files linearized with a standard reduction pipeline (Pipeline Processing System, PPS) at the Survey Science Center (SSC, University of Leicester, UK).

Events spread at most in two contiguous pixels for PN (i.e. pattern $=0-4$ ) and in four contiguous pixels for MOS (i.e., pattern $=0-12$ ) have been selected. Event files were cleaned from bad pixels (hot pixels, events out of the field of view, etc.) and the soft proton flares.

In order to remove periods of unwanted high background level, we located the flares by analysing the light curves of the count rate at energies higher than $10 \mathrm{keV}$, where the X-ray source contribution is negligible. We rejected the time intervals when the count rate was higher than 11 ( 8 for 0212481101 observation) counts $\mathrm{s}^{-1}$ and 1.3 counts $\mathrm{s}^{-1}$ for the PN and MOS cameras, respectively. The source counts were extracted from a circular region with a 25 arcsec radius. The background counts were extracted from the nearest source-free region. The response 
Table 2. X-ray detection parameters.

\begin{tabular}{ccccccc}
\hline \hline obs.id. & UT Date & MJD $^{a}$ & EPIC & $t_{\text {int }}$ & \multicolumn{2}{c}{$\left(\text { counts s}^{-1}\right)^{b}$} \\
cam & $(\mathrm{s})$ & $(0.5-10 \mathrm{keV})$ & $(0.5-2 \mathrm{keV})$ \\
\hline 0212480301 & Feb. 18, 05 & 53419 & PN/MOS & $1.7 \times 10^{4}$ & 0.0162 & 0.0132 \\
0212481101 & Sep. 08, 05 & 53621 & PN & $1.3 \times 10^{4}$ & 0.0044 & 0.0027 \\
\hline
\end{tabular}

${ }^{a}$ MJD = modified Julian Date.

${ }^{b}$ Counts are for the PN camera and are background subtracted.

Table 3. Near-IR photometry of V1118 Ori.

\begin{tabular}{cccccc}
\hline \hline MJD $^{a}$ & $J$ & $H$ & $K$ & $J-H$ & $H-K$ \\
\hline $48260-49350^{b}$ & $12.21(1)$ & $11.29(1)$ & $10.49(1)$ & 0.92 & 0.80 \\
$49426^{c}$ & $8.52(5)$ & $8.04(4)$ & $7.94(3)$ & 0.48 & 0.10 \\
$51872^{d}$ & $12.64(2)$ & $11.51(3)$ & $10.85(2)$ & 1.13 & 0.66 \\
\hline 53449 & $11.16^{e}$ & - & 9.79 & - & - \\
53463 & 10.79 & - & 9.48 & - & - \\
53475 & 10.94 & - & 9.53 & - & - \\
53619 & 11.08 & - & 9.71 & - & - \\
53624 & 11.23 & 10.45 & 9.85 & 0.78 & 0.60 \\
\hline
\end{tabular}

${ }^{a}$ MJD = modified Julian Date.

${ }^{b}$ The authors (Hillenbrand et al. 1998) do not specify the date of the observation made during the period 1991-1993. The errors (in units of $0.01 \mathrm{mag}$ ) are given in parentheses.

${ }^{c}$ Data from García García et al. (1995).

${ }^{d}$ 2MASS observation.

${ }^{e}$ Errors of our photometry in all the three bands never exceed 0.02 mag.

and ancillary files were generated by the XMM-SAS tasks, RMFGEN and ARFGEN, respectively. The MOS1 and MOS2 spectra were combined together, and the spectra binned to have at least 20 counts per energy bin.

\section{Results and discussion}

\subsection{Near-IR imaging and photometry}

The photometric data are presented in Table 3. For the sake of completeness, we report the near-IR photometric data available in literature prior to the recent outburst in the first three lines of Table 3. The maximum brightness corresponds to the third outburst's active phase (García García et al. 1995) and the minimum to the most recent quiescent phase (2MASS All-Sky Catalog of Point Sources - Cutri et al. 2003). The $J, H$ and $K$ brightness decreases by 4.1, 3.5, and 2.9 mag, respectively; thus less and less pronounced variations occur, while the wavelength increases. The third available near-IR observation (Hillenbrand et al. 1998) was obtained during an almost quiescent phase and it agrees with the described trend. Our results (from the fourth to the last lines of Table 3), sample the slow decline after the recent outburst and substantially agree with the results of Audard et al. (2005).

Since the data given in Table 3 refer to different outburst and quiescence phases and are not taken simultaneously with the optical data collected from the literature, they should not be used to construct any significant SED. However, we can assume that maxima and minima of different epochs recur at similar flux levels, a circumstance that is reasonably confirmed by the past record of optical observations. Under this hypothesis we plot two SEDs in Fig. 1, obtained by considering the lowest and the highest values, respectively. To enlarge the SED frequency range as much as possible, we searched the IR archives (IRAS, ISO, MSX) for a mid- to far-infrared counterpart of V1118 Ori

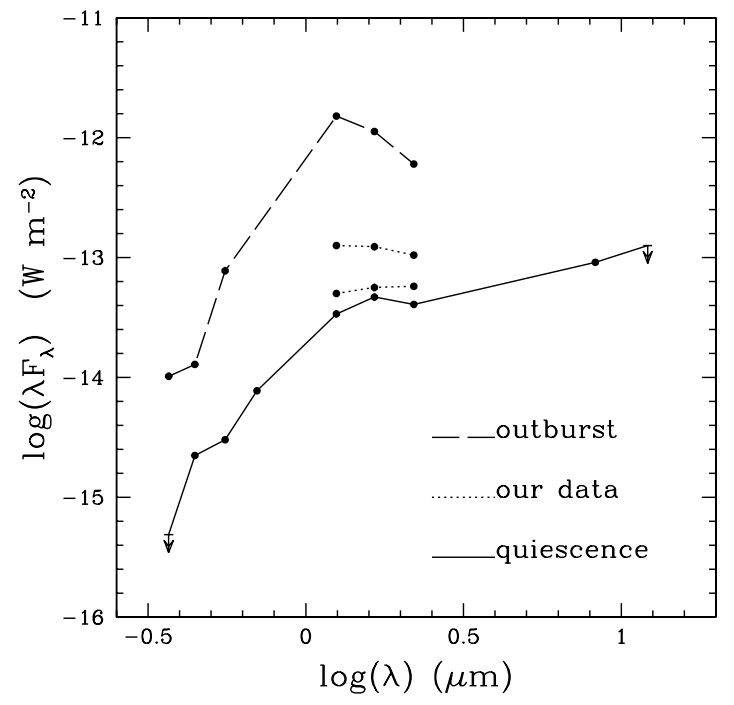

Fig. 1. Observed SEDs of V1118 Ori constructed with the literature and present paper IR data that correspond to different activity phases as indicated in the bottom right corner.

and we were able to detect a faint emission (at a $2 \sigma$ level) from a point-like source only in the MSX $A$-band at $8.28 \mu \mathrm{m}$, which corresponds to a flux density of $0.3 \mathrm{Jy}$, obtained during a quiescent phase. This value suffers from a certain degree of contamination, about $30 \%$, which is due not to the nearby bright star V372 Ori, but to the irregular diffuse emission characteristic of this sky area. In the MSX C-band (at $12.13 \mu \mathrm{m}$ ), only an upper limit of $0.5 \mathrm{Jy}$, corresponding to the closeby sky emission, can be derived. This MSX determination, although marginal, is quite important since permits us both to delineate the SED shape from the $U$ band to the mid-IR and to compute a reliable value of the bolometric luminosity $\left(L_{\mathrm{bol}}\right)$. The MSX detection was obtained during an inactive phase (July 1996), and as such, it has to be attributed only to the quiescent SED. We lack midIR detection during an active phase; however, the source is expected to increase its brightness at all wavelengths, as proven by Muzerolle et al. (2005). In fact, they reveal the outburst of V1647 Ori (whose EXor or FUor nature is still not ascertained) with the Spitzer Space Telescope and report a brightening in all the 3.6-70 $\mu \mathrm{m}$ range. By integrating the flux densities, we obtain $L_{\text {bol }}$ values of 1.4 (quiescence) and 25.4 (outburst) $L_{\odot}$, respectively. The latter has to be considered as a lower limit, since in the $L_{\text {bol }}$ (outburst) computation we used the same flux at $8.28 \mu \mathrm{m}$ ascribed to the inactive phase. These values indicate how the increase in brightness is indeed impressive, a factor of more than 18 , and suggest that the V1118 Ori luminosity $\left(<10 L_{\odot}\right)$ is more typical of the EXors than are the 5-50 times more luminous FUors.

The SEDs reported in Fig. 1 present different shapes: more peaked in the near infrared, looking like the $\mathrm{T}$ Tauri averaged 


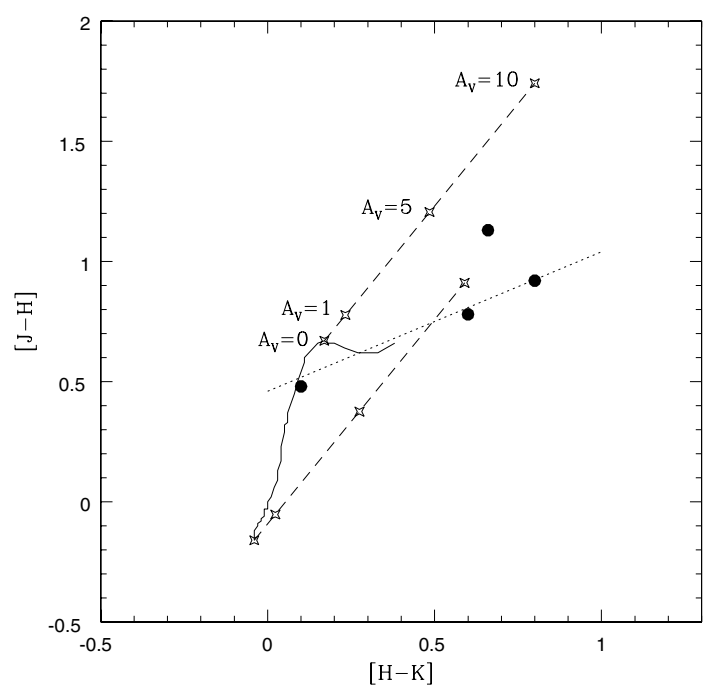

Fig. 2. Locations of V1118 Ori on a near-IR two-colours diagram in different epochs. The solid line marks the unreddened main sequence, whereas the dotted one is the locus pertaining to the $\mathrm{T}$ Tauri stars (Meyer et al. 1997). Dashed lines are the reddening law according to Rieke \& Lebofsky (1985); 10 mag intervals of $A_{\mathrm{V}}$ are indicated by crosses.

SED (D'Alessio et al. 1999), or rather flat, which resembles the one typical of the eruptive objects (FUors-EXors) (Kóspál et al. 2004; Muzerolle et al. 2005). All these shapes are characteristic of accreting T Tauri stars that indeed show variable SEDs in IR. The apparent steepening of the SED during the outburst is due to a single $J H K$ photometry and, as such, does not necessarily apply to all the outburst phases. Based on $J H K$ data plotted by Audard et al. (2005), a systematic variation in the SED slope with the activity phase does not seem recognizable. A systematic IR monitoring of repeated outbursts has to be accumulated to ascertain whether or not the SED is flattening while the source is progressively fading.

The same aspect can be discussed further by following how the source behaves in the $[J-H],[H-K]$ two-colour diagram given in Fig. 2. The locations of V1118 Ori, as detected in epochs of different levels of activity, indicate how the colours agree with those predicted for T Tauri stars by Meyer et al. (1997). Such predictions come from disk models with a range of accretion rates, inner disk radii, and viewing angles. We notice how the observed data are largely consistent with unreddened values, implying that in the majority of the cases the object is seen practically through a negligible extinction $\left(A_{\mathrm{V}} \simeq 0\right)$. In one phase, far from the outburst, it appears slightly redder and the same thing also happens in the optical band. The very similar case of the outburst recently studied over a large range of frequencies, namely V1647 Ori, behaves exactly in the same manner as V1118 Ori in the near-IR (Reipurth \& Aspin 2004; Vacca et al. 2004). With reference to Fig. 2 the observed shift in colours is compatible with an extinction value of $A_{\mathrm{V}} \sim 2 \mathrm{mag}$. Variable scattering by some cavity walls might contribute to make the emerging light bluer.

Several comparison stars are available within the 4.'4 SWIRCAM field; in particular one of them has remained stable within $0.01 \mathrm{mag}$ in $J H K$ during our entire monitoring period, so it is used to construct the near IR light curves in terms of differential photometry. A slightly downward trend is superposed on statistically meaningful variations (of about $0.2-0.3 \mathrm{mag}$ ) in the near IR on a shorter time scale (days). Such rapid variations
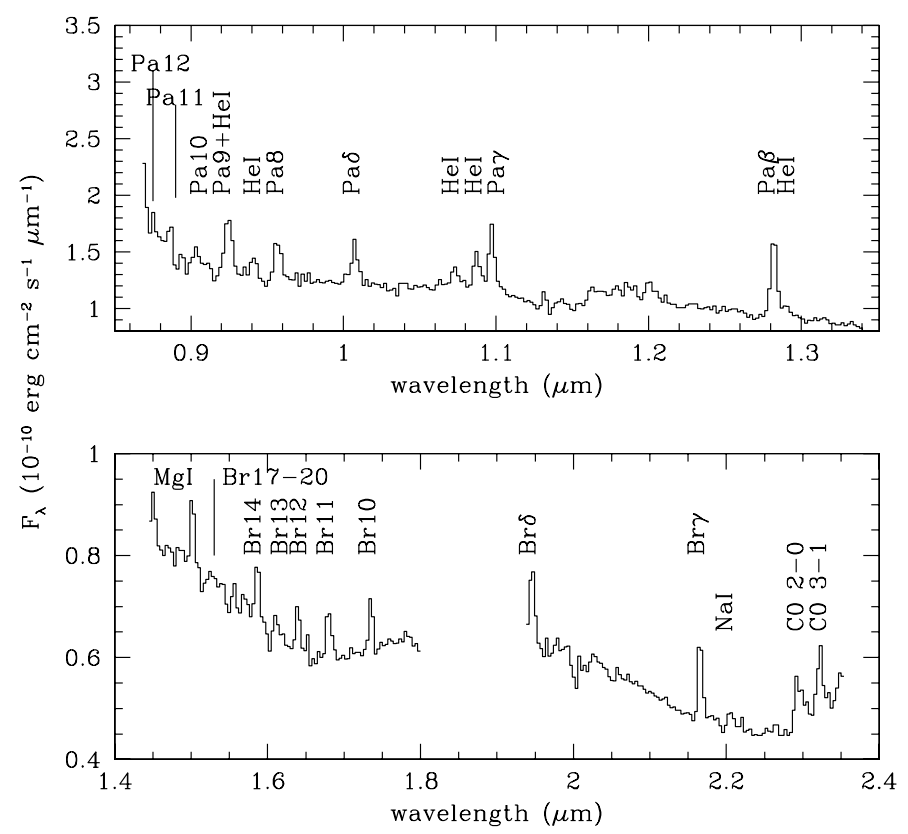

Fig. 3. Near-IR spectrum of V1118 Ori. The lines Pa 11 and Pa 12 are identified here, but they are neither listed in Table 4 nor used in our model, since their fluxes suffer large uncertainties due to the definition of a reliable local baseline.

detected in all the $J H K$ bands do not seem related to extinction variability, which should determine larger peak-to-peak variations at shorter wavelengths. This circumstance supports the idea that accretion processes are dynamical on several timescales: non steady accretion and rotational modulations occur from hours to weeks, while instabilities of the magneto-spherical structure and EXors-FUors events are characterised by longer timescales (months to years).

Our $\mathrm{H}_{2}$ image at $2.122 \mu \mathrm{m}$ shows no emission down to a level of $1.1 \times 10^{-14} \mathrm{erg} \mathrm{s}^{-1} \mathrm{~cm}^{-2}$ (at $3 \sigma$ ) within a $4.4 \times$ $4.4 \operatorname{arcmin}^{2}$ FoV. The $1-0 S(1) \mathrm{H}_{2}$ line at $2.122 \mu \mathrm{m}$ is one of the best tracers of the gas cooling after the passage of a shock front typically associated to jet structures emerging from a central object. Our sensitivity for revealing knots of $\mathrm{H}_{2}$ emission $\left(L_{\mathrm{H}_{2}}=\right.$ $\left.7 \times 10^{-5} L_{\odot}\right)$ would have allowed us to detect the faintest knots and jets discovered by Stanke et al. (2002) during their unbiased $\mathrm{H}_{2}$ survey of Orion. No other IR spectral features indicative of shock cooling (e.g. [Fe II] at 1.25 or $1.64 \mu \mathrm{m}$ ) are detected in our spectrum (see below, Sect. 3.2). Although the presence of an optical jet cannot be excluded, our observation agrees with the lack of collimated jets. This is quite a common feature in EXor and FUor objects that usually show powerful mass loss only in the form of isotropic winds.

\subsection{Near-IR spectroscopy}

During the recent slowly declining phase, we obtained the first near-IR spectrum of V1118 Ori depicted in Fig. 3 (see also Di Paola et al. 2005). The derived line fluxes are given in Table 4. It is an emission line spectrum dominated by the hydrogen recombination (Brackett and Paschen series), which signals the presence of ionized gas close to the star. Our spectral resolution does not allow us to reveal whether lines have P Cygni profiles. We also detected $\mathrm{Mg}$ I line emission at $1.503 \mu \mathrm{m}$, weaker emission features of $\mathrm{He} \mathrm{I}$, and a marginal (at $S / N=2$ ) emission of Na I at $2.208 \mu \mathrm{m}$. These atomic features are commonly found 
Table 4. Line emission fluxes of V1118 Ori.

\begin{tabular}{ccc}
\hline \hline $\begin{array}{c}\lambda_{\text {vac }} \\
\mu \mathrm{m})\end{array}$ & Ident. & $\begin{array}{c}F \pm \Delta F \\
\left(10^{-13} \mathrm{erg} \mathrm{s}^{-1} \mathrm{~cm}^{-2}\right)\end{array}$ \\
\hline 0.9017 & $\mathrm{~Pa} 10$ & $1.6 \pm 0.1$ \\
0.9231 & $\mathrm{~Pa} 9+\mathrm{He} \mathrm{I}$ & $3.1 \pm 0.1$ \\
0.9466 & $\mathrm{He} \mathrm{I}$ & $0.8 \pm 0.1$ \\
0.9548 & $\mathrm{~Pa} 8$ & $2.3 \pm 0.2$ \\
1.0052 & $\mathrm{~Pa} \delta$ & $2.2 \pm 0.2$ \\
1.0670 & $\mathrm{He} \mathrm{I}$ & $1.2 \pm 0.3$ \\
1.0832 & $\mathrm{He} \mathrm{I}$ & $1.9 \pm 0.2$ \\
1.0941 & $\mathrm{~Pa} \gamma$ & $2.5 \pm 0.2$ \\
1.2822 & $\mathrm{~Pa} \beta$ & $4.1 \pm 0.1$ \\
1.2850 & $\mathrm{He} \mathrm{I}$ & $1.0 \pm 0.1$ \\
1.5031 & $\mathrm{Mg} \mathrm{I}$ & $0.8 \pm 0.2$ \\
1.5885 & $\mathrm{Br} 14$ & $0.8 \pm 0.2$ \\
1.6114 & $\mathrm{Br} 13$ & $0.5 \pm 0.2$ \\
1.6412 & $\mathrm{Br} 12$ & $0.6 \pm 0.2$ \\
1.6811 & $\mathrm{Br} 11$ & $0.9 \pm 0.3$ \\
1.7367 & $\mathrm{Br} 10$ & $0.8 \pm 0.2$ \\
1.9451 & $\mathrm{Br} \delta$ & $1.0 \pm 0.2$ \\
2.1661 & $\mathrm{Br} \gamma$ & $1.2 \pm 0.2$ \\
2.2084 & $\mathrm{Na} \mathrm{I}$ & $0.4 \pm 0.2$ \\
2.2935 & $\mathrm{CO} 2-0$ & $1.2 \pm 0.4$ \\
2.3227 & $\mathrm{CO} 3-1$ & $1.3 \pm 0.3$ \\
\hline
\end{tabular}

in the near-IR spectra of T Tauri stars and younger objects. In particular, $\mathrm{Mg}$ I and $\mathrm{NaI}$ emission lines have been recently revealed in another erupting object, namely V1647 Ori (Reipurth \& Aspin 2004; Vacca et al. 2004), where He I is however present in absorption. We note that a $\mathrm{NaI}$ line has been also detected, but in absorption (Herbig et al. 2001), in the near-IR spectrum of EX Lup, the prototype of the EXor class. These circumstances suggest that an IR spectroscopic database of eruptive variables, large enough to cover different activity phases, has to be built up in order to evaluate how possible similarities among EXors can be related to the dominant mechanism(s) of gas excitation and cooling. Molecular hydrogen contributions are absent and only the 1-0 $S$ (1) rovibrational line at $2.122 \mu \mathrm{m}$ is marginally recognizable at a $1 \sigma$ level; such a spectroscopic circumstance confirms the lack of any shock evidence also indicated by $\mathrm{H}_{2}$ imaging (Sect. 3.1). The observed spectrum is much more similar to that of an accreting T Tauri star (Greene \& Lada 1996) than the FUor ones. All these have spectra always dominated by absorption lines (Reipurth \& Aspin 1997), apart a couple of exceptions.

We have checked that no contamination exists due to the HII region associated to the nearby bright star V372 Ori. In Table 4 the identified lines are given along with the measured fluxes. The associated uncertainty refers to the rms of the local baseline.

The CO overtone emission $v=2-0, v=3-1$ is clearly detected; the same occurs in several young stellar objects (Carr 1989), although in the majority of the FUor the CO bands are revealed in absorption (Hartmann et al. 2004). In all cases, $\mathrm{CO}$ emission is seen together with $\mathrm{Br} \gamma$ line emission, but these two features probably come from different volumes of gas. At temperatures of about $4000 \mathrm{~K}, \mathrm{CO}$ is dissociated and molecular hydrogen is dissociated by collisions for values higher than $3000 \mathrm{~K}$. However, for density values higher than $10^{7} \mathrm{~cm}^{-3}$ and in the presence of $\mathrm{H}_{2}, \mathrm{CO}$ dominates the cooling (Scoville et al. 1980). Therefore the CO bands are specific probes of the circumstellar portions where the gas is relatively warm at high densities. Carr (1989) has quantitatively investigated two scenarios that account for the observed $\mathrm{CO}$ emission: an accretion disk and a neutral stellar wind with velocities of $100-300 \mathrm{~km} \mathrm{~s}^{-1}$,
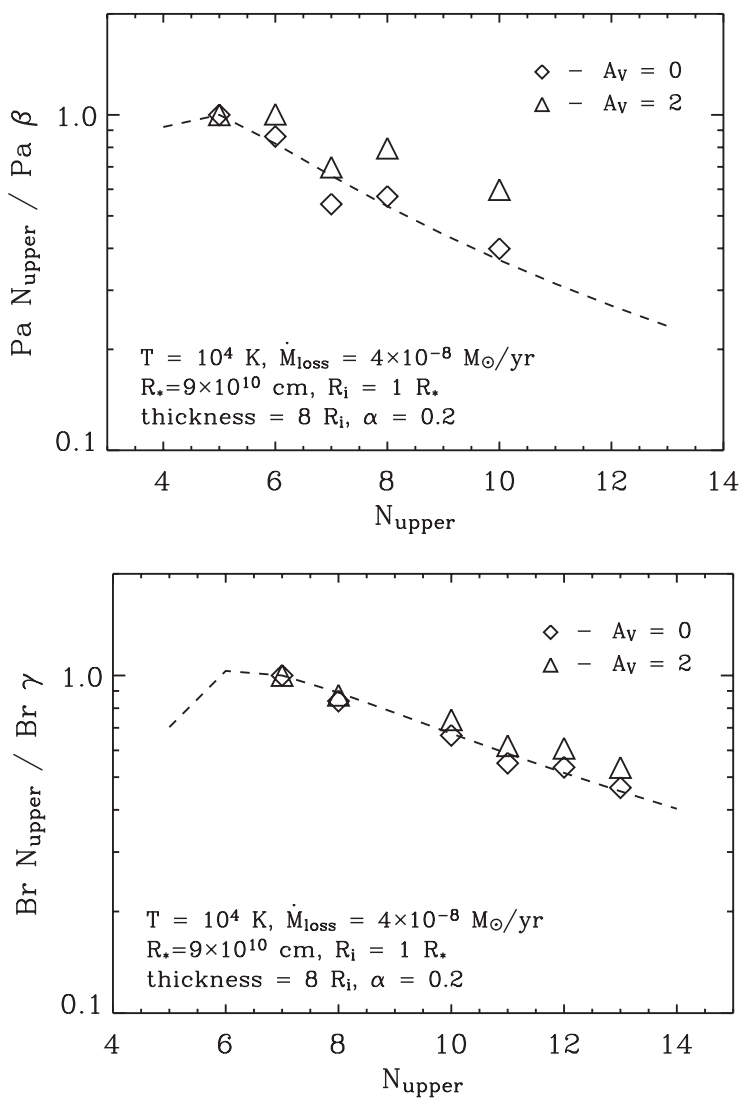

Fig. 4. Top panel: line ratios of the Paschen series with respect to the $\mathrm{Pa} \beta$ line. Diamonds and triangles refer to our observations when undereddened $\left(A_{\mathrm{V}}=0\right)$ and extinction-corrected $\left(A_{\mathrm{V}}=2\right)$, respectively. The dashed line represents the model line ratios. The relevant fit parameters are reported at the top of the panel. Bottom panel: the same, but line ratios refer to the Brackett series with respect to the $\mathrm{Br} \gamma$ line.

where the gas is assumed to be cool $(\sim 3000 \mathrm{~K})$. In this case, which agrees with our observations (see below this section), he provides model predictions for deriving the mass loss rate from the CO (2-0) luminosity (his Fig. 8). The CO flux, calculated in the $2-0$ band by integrating from 2.29 and $2.32 \mu \mathrm{m}$ after continuum subtraction, provides a $\mathrm{CO}$ luminosity $L_{\mathrm{CO}}$ that is equal to $6.3 \times 10^{-5} L_{\odot}$ and consequently a mass loss value in the range (3-8) $\times 10^{-7} M_{\odot} \mathrm{yr}^{-1}$ can be derived. The uncertainty range accounts for the varying model parameters.

The observed HI line emission has been compared with a wind model (Nisini et al. 2004) that considers a spherically symmetric and partially ionized envelope with a constant rate of mass loss $\left(\dot{M}=4 \pi r^{2} \rho(r) v(r)\right)$. The emitting gas is assumed to be in LTE and the adopted gas velocity law is:

$v(r)=v_{i}+\left(v_{\max }-v_{i}\right)\left[1-\left(R_{*} / R\right)^{\alpha}\right]$

where $v_{i}$ and $v_{\max }$ are the initial and maximum wind velocities, respectively, while $R_{*}$ is the stellar radius. The best fit to the data points is obtained for the following set of parameters: gas temperature of $10^{4} \mathrm{~K}, v_{i}=20 \mathrm{~km} \mathrm{~s}^{-1}, v_{\max }=200 \mathrm{~km} \mathrm{~s}^{-1}$, the envelope's internal radius $R_{i}=1 R_{*}$, envelope thickness equal to $8 R_{*}$, mass loss rate $\dot{M}=4 \times 10^{-8} M_{\odot} \mathrm{yr}^{-1}$. An important parameter of the model is the extinction value $\left(A_{\mathrm{V}}\right)$ for which the line fluxes need to be corrected. From analysing the position of the object in the two-colour diagram (Sect. 3.1), we have assumed $A_{\mathrm{V}}=$ 0 , a value very consistent with our observations. Figure 4 shows the line ratios of the Brackett and Paschen series with respect 
to the $\mathrm{Br} \gamma$ and $\mathrm{Pa} \beta$, respectively, along with the best-fit model, whose parameters are also indicated. In addition, the model predicts a flux of $1.3 \times 10^{-13} \mathrm{erg} \mathrm{s}^{-1} \mathrm{~cm}^{-2}$ for the $\mathrm{Br} \gamma$, practically equal to the observed one $\left(1.2 \times 10^{-13} \mathrm{erg} \mathrm{s}^{-1} \mathrm{~cm}^{-2}\right.$, see Table 4). The effects of introducing a different extinction value $\left(A_{\mathrm{V}}=2\right.$, namely the maximum allowed by the observations) are also depicted in Fig. 4.

Finally, by ratioing the mass loss rates derived from the HI recombination $\left(4 \times 10^{-8} M_{\odot} \mathrm{yr}^{-1}\right)$ and the $\mathrm{CO}$ emission $\left((3-8) \times 10^{-7} M_{\odot} \mathrm{yr}^{-1}\right)$, respectively, a ionization fraction of about $0.1-0.2$ can be derived. This value is largely consistent with those typical of active T Tauri environments (Greene \& Lada 1996).

To evaluate the uncertainties on the derived parameters and to check the sensitivity of our model, we computed the range of variation for each input parameter that is allowed to eventually provide line flux predictions comparable (within a 50\% extent) to the observed values. Such analysis indicates that gas temperature is one of the less sensitive parameters: variations between $5000 \mathrm{~K}$ and $15000 \mathrm{~K}$ do not affect the fit significantly. Conversely, other parameters are quite critical and their variability ranges are consequently rather narrow: $\dot{M}=4 \pm 2 \times$ $10^{-8} M_{\odot} \mathrm{yr}^{-1}, R_{i}=1-2 R_{*}$, thickness $8 \pm_{2}^{4} R_{*}$. Finally we note that no realistic solution can be found by using $A_{\mathrm{V}}=2$ mag, as this would imply an $\dot{M}$ increase of about $50 \%$ and a predicted $\mathrm{Br} \gamma$ flux exceeding the observed value by more than one order of magnitude.

\section{3. $X$-ray properties}

The XMM data were modeled using the XPEC spectral analysis package. The first observation (see Table 2) was first fitted over the 0.5 to $10 \mathrm{keV}$ range with an almost unabsorbed two-temperature MEKAL thermal model (Mewe et al. 1995), which minimizes the $\chi^{2}$. The solar metal abundance has been adopted for Orion (Esteban et al. 1998); however, a trial with a value half solar provided no significant improvement in the $\chi^{2}$ value. Figure 5 (top panel) compares the observed spectra with the best fit model. The derived parameters are given in the first line of Table 5. By looking at the fit, the suspicion could arise that a background contamination affects the data at higher energies than 3-4 keV. Therefore, as a second attempt, we fitted only the data points up to $3.5 \mathrm{keV}$. Following the analysis of Audard et al. (2005), we fitted these (2005 February) data with a single temperature, obtaining the result given in Fig. 5 (middle panel), along with the parameters reported in the second line of Table 5; these parameters agree substantially with the Audard et al. results. We also investigated the possibility of fitting the data with a two-temperature model obtaining the result plotted in Fig. 5 (bottom panel), and the parameters given in the third line of Table 5. The two-temperature fit is as acceptable as the single temperature (see the residuals behaviour in Fig. 5 (bottom panel)) and has an $N_{\mathrm{H}}$ value that agrees better with a rather unobscured line of sight. The circumstance that a good fit can be obtained with two-temperature values (about 30 and $7 \mathrm{MK}$ ), similar to those able to account for the X-ray emission as detected prior the outburst (Audard et al. 2005), confirms that the X-ray emission of V1118 Ori is practically unaffected by accretion events, since originated in the corona. Thus, the eventual disappearance of a high temperature component of the coronal emission should occur with a given delay with respect to the outburst. The second spectrum reported in Table 2 was obtained in Sep. 2005 (i.e. seven months later). Although this latter X-ray spectrum is integrated as long as the former, it
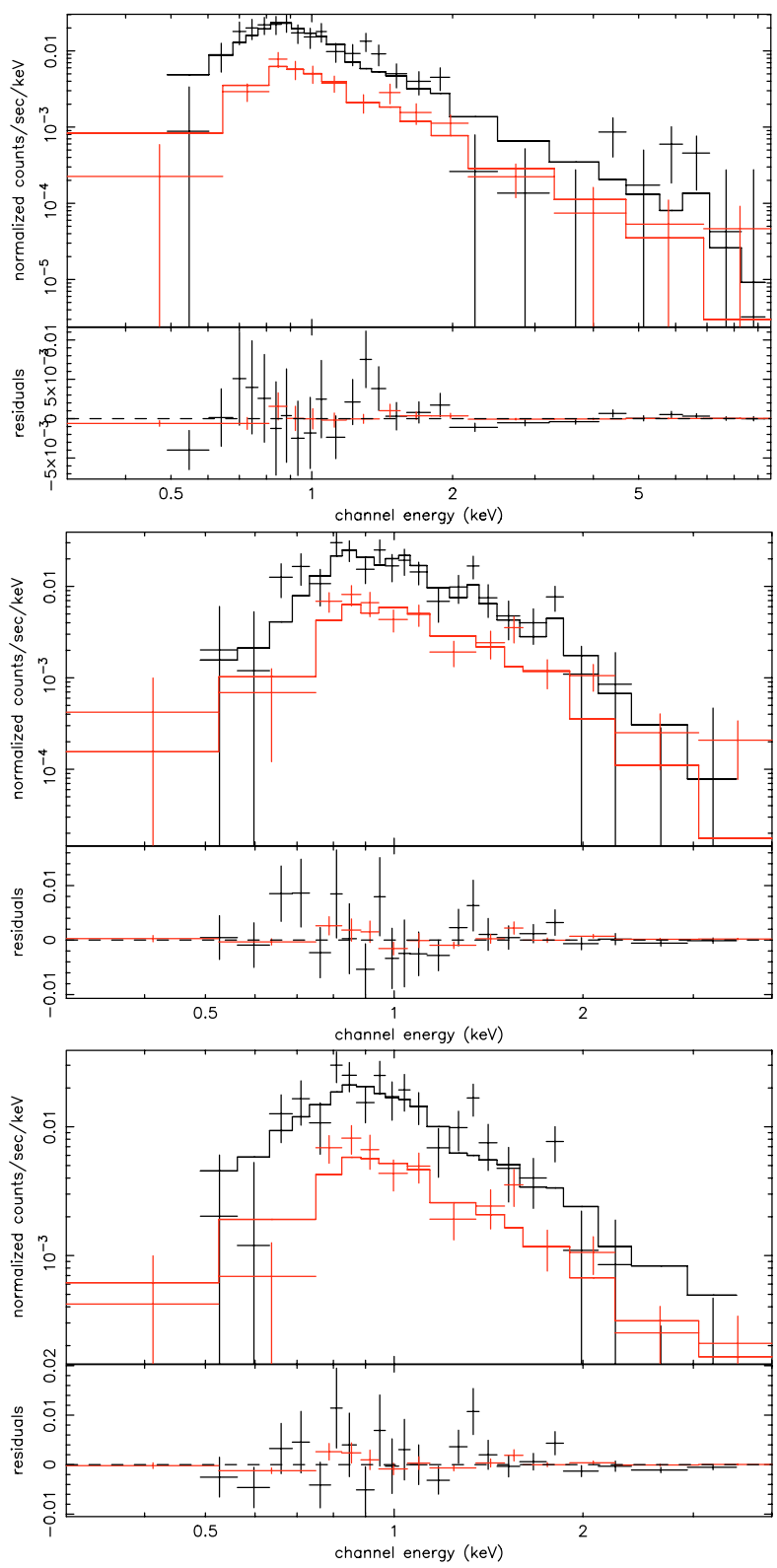

Fig. 5. Top panel: X-ray spectrum (0.5-10 keV - February 2005) of V1118 Ori observed with the EPIC PN and MOS detectors on board XMM. A double-temperature thermal model has been adopted to fit the data (see text). The residuals computed in each bin are also reported. Middle panel: as above, where the $0.5-3.5 \mathrm{keV}$ portion is fitted with a single-temperature model. Bottom panel: as above, where the $0.5-3.5 \mathrm{keV}$ portion is fitted with a double-temperature model.

appears intrinsically weaker, and a decent fit can be obtained only with a single temperature (of about $10 \mathrm{MK}$ ) and by leaving the absorbing column density $\left(N_{\mathrm{H}}\right)$ as a free parameter (fourth line of Table 5). However, the best fit value, corresponding to a visible extinction $A_{\mathrm{V}}<2$ (see Table 5) is consistent with the earlier observation $\left(A_{\mathrm{V}}=1\right)$. This fact is quite important since it proves that the X-ray fading is real and not due to any intervening extinction effect. Remember that the near-IR spectrum, taken in coincidental simultaneity (just 3 days later), could also be fitted only by assuming $A_{\mathrm{V}} \simeq 0$. The small difference between the extinction value derived from the X-ray measurement by assuming a gas-to-dust ratio typical of the interstellar medium and that derived from the simultaneously taken near IR spectrum, confirms the suggestion that the gas-to-dust ratio 
Table 5. X-ray derived parameters.

\begin{tabular}{cccccccccc}
\hline \hline UT Date & $\chi^{2}$ & d.o.f. & $\begin{array}{c}N_{\mathrm{H}} \\
\left(10^{22} \mathrm{~cm}^{-2}\right)\end{array}$ & $\begin{array}{c}k T_{1} \\
(\mathrm{keV})\end{array}$ & $\begin{array}{c}E T_{2} \\
\left(10^{52} \mathrm{~cm}^{-3}\right)\end{array}$ & $\begin{array}{c}E M^{a} \\
(0.5-10 \mathrm{keV})\end{array}$ & $\begin{array}{c}\text { Flux }\left(10^{-14} \mathrm{erg} \mathrm{s}^{-1} \mathrm{~cm}^{-2}\right) \\
(0.5-3.5 \mathrm{keV})\end{array}$ \\
\hline Feb. 18, 05 & 35.7 & 35 & $0.19^{b}$ & $2.8 \pm_{1.1}^{3.8}$ & $0.6 \pm 0.1$ & $5 \pm 1$ & $2.5 \pm 0.5$ & $4.0\left(5.8^{c}\right)$ & $2.4\left(4.1^{c}\right)$ \\
Feb. 18, 05 & 36.8 & 33 & $0.8 \pm 0.1$ & - & $0.52 \pm_{0.10}^{0.07}$ & - & $24 \pm_{8}^{12}$ & - & $2.2\left(23.7^{c}\right)$ \\
Feb. 18, 05 & 41.6 & 32 & $0.19^{b}$ & $2.5 \pm_{0.8}^{2.8}$ & $0.6 \pm 0.1$ & $5 \pm 1$ & $2.0 \pm_{0.2}^{0.7}$ & - & $2.2\left(3.8^{c}\right)$ \\
Sep. 08, 05 & 6.2 & 4 & $<0.9^{d}$ & - & $1.0 \pm_{0.6}^{1.1}$ & - & $<12^{e}$ & - & $0.6\left(1.1^{c}\right)$ \\
\hline
\end{tabular}

${ }^{a}$ The emission measure $(E M)$ is derived for each temperature component from the fit parameter $K$, through the relationship $E M=\mathrm{K} \times 10^{14} 4 \pi d^{2}$, where $d$ is the source's distance (in $\mathrm{cm}$ ).

${ }^{b}$ Frozen parameter.

${ }^{c}$ Unabsorbed flux.

${ }^{d}$ The best fit value corresponds to $N_{\mathrm{H}}=0.4 \times 10^{22} \mathrm{~cm}^{-2}$.

${ }^{e}$ The best fit value corresponds to $E M=2.5 \times 10^{52} \mathrm{~cm}^{-3}$.

The quoted errors correspond to a $90 \%$ confidence level.

Table 6. V1118 Ori derived parameters.

\begin{tabular}{ccc}
\hline \hline Parameter ${ }^{a}$ & & Value \\
\hline Distance & $d$ & $460 \mathrm{pc}$ \\
Spectral Type & & Mle \\
Stellar radius & $R_{*}$ & $1.29 R_{\odot}$ \\
\hline Bolometric luminosity & $L_{\mathrm{bol}}$ & $1.4 L_{\odot}(\mathrm{min})$ \\
& & $25.4 L_{\odot}(\max )$ \\
X-ray luminosity & $L_{\mathrm{X}}(0.5-3.5 \mathrm{keV})$ & $0.6-2.2 \times 10^{-4} L_{\odot}$ \\
Plasma temperature & $T$ & $7-11 \times 10^{6} \mathrm{~K}$ \\
Visual extinction & $A_{\mathrm{V}}$ & $0-2 \mathrm{mag}^{-1}$ \\
Wind velocity & $v_{\max }$ & $200 \mathrm{~km} \mathrm{~s}^{-1}$ \\
Mass loss rate & $\dot{M}$ & $4 \pm 2 \times 10^{-8} M_{\odot} \mathrm{yr}^{-1}$ (ionized) \\
& & $3-8 \times 10^{-7} M_{\odot} \mathrm{yr}^{-1}$ (neutral) \\
Ionization fraction & & $0.1-0.2$ \\
Envelope size & & $8 \pm_{2}^{4} R_{*}$ \\
\hline
\end{tabular}

${ }^{a}$ The first three lines of the table list literature parameters, while the rest are derived in the present work.

near the young stars could be higher than interstellar (Kastner et al. 2004b). The Sep. 2005 observation corresponds to $L_{X}(0.5-$ $3.5 \mathrm{keV})=0.6 \times 10^{-4} L_{\odot}=2.7 \times 10^{29} \mathrm{erg} \mathrm{s}^{-1}$; assuming that the true stellar luminosity ranges between 0.5 and $1 L_{\odot}$, then $\log \left(L_{\mathrm{X}} / L_{\mathrm{bol}}\right)>-4.2$, which means the star is much more active than our Sun (for which $\log \left(L_{\mathrm{X}} / L_{\mathrm{bol}}\right) \sim-6.5$ ).

By examining the data in Table 5, we note: $i$ ) a real fading of about a factor of 4 has occurred in the X-ray emission $(0.5-3.5 \mathrm{keV})$ between February and September 2005; and ii) the existence of a temperature component of about $7 \mathrm{MK}$ during the active phases, interpreted as a coronal signature (Audard et al. 2005), is a persistent feature even in the post outburst phase, while the X-ray emission of the object begins to decline. Recently, Preibisch et al. (2005) have presented their survey (named COUP) of nearly $600 \mathrm{X}$-ray emitting T Tauri stars in Orion and interpreted the widespread existence of a common temperature around $10 \mathrm{MK}$, or less, as a real feature of the coronal temperature distribution. Our data agree fully with their findings, confirming the active T Tauri nature of V1118 Ori. Attributing the fairly constant temperature component, detected even at a lower level of X-ray emission, to the coronal activity contrasts with the interpretation by Grosso et al. (2005) of the recent eruption of V1647 Ori (Kastner et al. 2004b); however, the inconsistencies pertaining to the long-standing debate on the dominant X-ray mechanism in YSOs are discussed by Audard et al. (2005).
The detected fading could be interpreted in the context of the magnetically active stars that show a significant X-ray variability (e.g. Feigelson \& Montmerle 1999), but its timing with an optical-IR post-outburst phase, if not completely fortuitous, can still support a direct connection: a sort of modulation over the coronal mechanism that tends to reduce the X-ray emission. Indeed, according to Preibisch et al. (2005), the accretion events do reduce the X-ray activity; we can only say that this effect cannot be explained as due to intervening extinction, but to some other effects (e.g. a weaker dynamo action related to the magnetic disk locking).

\section{The emerging picture and concluding remarks}

In this section we summarise the results presented above by trying to delineate a coherent picture for V1118 Ori. First, in Table 6 the relevant information derived in the present work is listed along with some data from the literature.

V1118 Ori appears to be an accreting young object that undergoes quasi periodic outbursts whose monitoring, so far conducted only in the visual band, has also been started in the near IR and X-ray bands. The first marginal detection in the mid-IR (near $8 \mu \mathrm{m}$ ) is also provided. Our observations largely confirm its EXor nature: its bolometric luminosity increases from $1.4 L_{\odot}$ to more than $25 L_{\odot}$ passing from inactive to active phases.

V1118 Ori presents a SED typical of an accreting T Tauri star and also its near-IR spectrum strictly resembles that of T Tauri, showing strong $\mathrm{HI}, \mathrm{He} \mathrm{I}$, and $\mathrm{CO}$ features in emission. The near-IR colours do not seem to undergo extinction variations and are always compatible with low $A_{\mathrm{V}}$ values ( $0-2 \mathrm{mag}$ ). Beside the accretion disk, the star has a quite compact and partially ionized circumstellar envelope created by the mass loss in stellar winds that are very likely accretion-generated. V1118 Ori is presumably in a late stage of its pre-main sequence evolution and this is likely the reason of its unobscured appearance. The lack of any collimated outflow driven by the source further supports this hypothesis. A large part of the relevant physics of V1118 Ori originates in its accretion disk. By studying its X-ray properties, a coronal origin is confirmed as responsible for the high energy emission with some influence by accretion. The parameters derived by X-ray observations (plasma temperature and ratio $L_{\mathrm{X}} / L_{\mathrm{bol}}$ ) all agree with those obtained for active T Tauri stars.

All the observational evidence presented in this paper shows the similarity of V1118 Ori to low-luminosity unobscured T Tauri stars. This behaviour suggests that: (i) the EXor 
evolutionary stage appears to follow the FUor one, more likely than being a less evident manifestation of the same phase; and (ii) the scanty number of known EXor could be simply due to the difficulty of performing an accurate multi-frequency monitoring of all the active T Tauri stars.

We will continue our IR monitoring program by also extending the investigation to shorter timescales (hours, days). Indeed, significant clues of rapid variations are already presented here (Sect. 3.1), but large databases have to be accumulated to properly study to what extent the accretion processes are dynamical on several timescales.

Acknowledgements. The authors would like to thank the referee Marc Audard for his constructive comments on different aspects of this paper.

\section{References}

Audard, M., Güdel, M., Skinner, S. L., et al. 2005, ApJ, 635, L81

Carr, J. S. 1989, ApJ, 345, 522

Chanal, M. 1983, IAUC, 3763

Cutri, R. M., Strutskie, M. F., Van Dyk, S., et al. 2003, Explanatory Supplement to the 2MASS All Sky Data Release (Pasadena: Caltech)

D'Alessio, F., et al. 2000, Proc. of the SPIE Symp. on Astronomical Telescopes and Instrumentation, ed. M. Iye, \& A. F. M. Moorwood, 4008, 748

D’Alessio, P., Calvet, N., Hartmann, L., Lizano, S., \& Cantó, J. 1999, ApJ, 527, 893

Di Paola, A., Lorenzetti, D., Calzoletti, L., et al. 2005, ATel, 619

Esteban, C., Peimbert, M., Torres-Peimbert, S., \& Escalante, V. 1998, MNRAS, 298, 185

Feigelson, E. D., \& Montmerle, T. 1999, ARA\&A, 37, 363

García García, J., Mampaso, A., \& Parsamian, E. S. 1995, Information Bulletin of Variable Stars, 4268

García García, J., \& Parsamian, E. S. 2000, Information Bulletin of Variable Stars, 4925
Gasparian, L. G., Melkonian, A. S., Ohanian, G. B., \& Parsamian, E. S. 1990, Flare Stars in Star Clusters, Associations, and the Solar Vicinity Proc. 137th Symposium of the International Astronomical Union, Byurakan [Armenia], USSR, October 23-27, 1989, ed. L. V. Mirzoyan, B. R. Pettersen, \& M. K. Tsvetkov (Dordrecht, The Netherlands, Boston, MA: Publ. Kluwer Academic)

Greene, T. P., \& Lada, C. J. 1996, AJ, 112, 2184

Grosso, N., Kastner, J. H., Ozawa, H., et al. 2005, A\&A, 438, 159

Hartmann, L., Hinkle, K., \& Calvet, N. 2004, ApJ, 609, 906

Hartmann, L., Kenyon, S., \& Hartigan, P. 2003, Protostars and Planets III, ed. E. H. Levy, \& J. I. Lunine, 497

Hayakawa, T., Ueda, T., Uemura, M., et al. 1998, Information Bulletin of Variable Stars, 4615

Herbig, G. H. 1977, ApJ, 217, 693

Herbig, G. H. 1989, Proc. of the ESO Workshop on Low Mass Star Formation and Pre-Main Sequence Objects, ed. B. Reipurth, 233

Hillenbrand, L. A., Strom, S. E., Calvet, N., et al. 1998, AJ, 620, L107

Jansen, F., Lumb, D., Altieri, B., et al. 2001, A\&A, 365, L1

Kastner, J. H., Huenemoerder, D. P., Schultz, N. S., et al. 2004a, ApJ, 605, L49

Kastner, J. H., Richmond, M., Grosso, N., et al. 2004b, Nature, 430, 429

Kóspál, Á., Ábrahám, P., \& Csizmadia, Sz. 2004, Baltic Astron., 13, 518

Meyer, M. R., Calvet, N., \& Hillenbrand, L. A. 1997, AJ, 114, 288

Mewe, R., Kaastra, J. S., \& Liedahl, D. A. 1995, Legacy, 6, 16 http://heasarc.gsfc.nasa.gov/docs/journal/meka6.html

Muzerolle, J., Megeath, S. T., Flaherty, K. M., et al. 2005, ApJ, 116, 1816

Nisini, B., Antoniucci, S., \& Giannini, T. 2004, A\&A, 421, 187

Parsamian, E. S., \& Gasparian, K. G. 1987, Astrophysics, 27, 598

Parsamian, E. S., Ibragimov, M. A., Ohanian, G. B., \& Gasparian, K. G. 1993, Astrophysics, 36, 23

Paul, C., Kroll, P., \& Lehmann, T. 1995, Lect. Notes Phys., 151, 229

Preibisch, T., Kim, Y.-C., Favata, F., et al. 2005, ApJ, in press

Reipurth, B., \& Aspin, C. 1997, AJ, 114, 2700

Reipurth, B., \& Aspin, C. 2004, ApJ, 606, L119

Rieke, G. H., \& Lebofsky, M. J. 1985, ApJ, 288, 618

Scoville, N. Z., Krotkov, R., \& Wang, D. 1980, ApJ, 240, 929

Stanke, T., McCaughrean, M. J., \& Zinnecker, H. 2002, A\&A, 392, 239

Strüder, L., Briel, U., Dennerl, K., et al. 2001, A\&A, 365, L18

Turner, M. J. L., Abbey, A., Arnaud, M., et al. 2001, A\&A, 365, L27

Vacca, W. D., Cushing, M. C., \& Simon, T. 2004, ApJ, 609, L29

Williams, P., \& Heathcote, N. S. W. 2005, IAUC, 8460 